\title{
Riesgo sistemático en el mercado mexicano de capitales: un caso de segmentación parcial ${ }^{1}$
}

\author{
Francisco López Herrera*
}

\section{Resumen}

En este documento se presentan los resultados obtenidos mediante la estimación de un modelo econométrico para estudiar la dinámica de la prima de riesgo del mercado mexicano de capitales. Las variables explicativas son los factores de riesgo mostrados por López y Ortiz (2005), construidos con base en variables económicas cuya importancia para explicar el riesgo sistemático ha sido identificada en la literatura teórica y en la derivada de estudios empíricos para el caso mexicano. En el análisis que se presenta en el documento se consideran también la relación del mercado mexicano con el mercado mundial de capitales y eventos importantes para el mercado mexicano de valores. Además de la evidencia de que los factores analizados son relevantes para explicar la prima de riesgo en México, se encuentra que la liberalización financiera, el Tratado de Libre Comercio de América del Norte (TLCAN) y el proceso de estabilización de la economía mexicana mediante el pacto y las políticas sucedáneas han tenido efectos significativos también. Asimismo, se encuentra evidencia de segmentación parcial del mercado mexicano de capitales con respecto del mundial.

Palabras clave: valuación de activos, modelos multifactoriales, prima de riesgo, riesgo sistemático y variables económicas, integración-segmentación de mercados de capitales.

1 Se agradecen los comentarios y sugerencias de los miembros del Seminario de Investigadores de la División de Investigación, FCA, UNAM, así como de los asistentes al IX Foro de Investigación, FCA-ANFECA, efectuado en Ciudad Universitaria en octubre de 2004. Se hace también un agradecimiento especial a los comentarios y observaciones hechas por dos árbitros anónimos. Naturalmente, los errores y omisiones que aún persistan son responsabilidad exclusiva del autor.

* Investigador de la División de Investigación de la Facultad de Contaduría y Administración, UNAM. Correo electrónico: francisco_lopez_herrera@yahoo.com.mx 


\section{Introducción}

Según Errunza y Miller (2000), hacia finales de la Segunda Guerra Mundial, $N_{\text {los }}$ mercados de capitales alrededor del mundo se caracterizaban por la existencia de numerosas barreras a los flujos de capital pues, además de las altas cargas impositivas y los elevados costos de transacción existentes, también había restricciones explícitas sobre la propiedad de activos locales por extranjeros, así como para la movilidad del capital y las operaciones cambiarias. Además, se observaba un menor grado de desarrollo de los mercados accionarios, con baja liquidez, regulaciones inadecuadas y requisitos laxos para la revelación de la información. Esta situación dio como resultado lo que Errunza (1979) de nomina la supresión del portafolio. Como resultado de la supresión del portafolio, las fuentes primarias de financiamiento para las empresas eran sus propios recursos, el crédito bancario y el financiamiento del grupo empresarial al que pertenecían. Sólo el gobierno, las empresas paraestatales y las grandes empresas tenían acceso a los mercados de capitales, fenómeno observado principalmente en las economías ahora denominadas emergentes. Los mercados nacionales de capitales eran pequeños, con muy poca actividad y altamente segmentados, pues la mayoría de los activos domésticos estaban en manos de inversionistas locales. Estas condiciones imponían a las empresas un costo de capital demasiado alto.

Durante la década de los años setenta del siglo pasado, varios países desarrollados, así como también algunos países en vías de desarrollo, como por ejemplo Argentina, Brasil y Chile, reformaron sus economías y liberalizaron sus sistemas financieros. Como consecuencia de la llamada crisis de la deuda del Tercer Mundo, los países deudores se encontraron sin acceso a las fuentes de financiamiento externo que requerían para promover su desarrollo, por lo que para enfrentar esa restricción impulsaron reformas a su economía, abriéndose al comercio internacional y a las inversiones extranjeras de portafolio para atraer capitales externos. Esos países han retirado diversos obstáculos a la inversión internacional de portafolios atrayendo una gran cuantía de flujos externos a sus mercados de valores, considerados como emergentes. Después del derrumbe del bloque socialista las economías en transición del socialismo al capitalismo se sumaron al proceso de apertura económica y liberalización financiera. 
Riesgo sistemático en el mercado mexicano de

capitales: un caso de segmentación parcial

Incluso China, formalmente aún bajo un régimen socialista, ha establecido mecanismos de apertura y liberalización de sus mercados financieros. Como consecuencia de la liberalización financiera se ha observado que a pesar del mayor crecimiento de la inversión extranjera directa entre 1990 y 2000 en comparación con la de portafolio, ésta fue mayor de 1991 a 1994 y representó casi el $43 \%$ del total de los flujos durante ese periodo de once años. Márquez, Islas y Venegas (2003) encuentran que entre 1980 y 1997 en el nivel mundial los flujos de inversión de portafolio crecieron casi 25 veces, en tanto que los flujos de inversión extranjera directa en todo el mundo lo hicieron únicamente 12 veces, siendo a partir de 1984 cuando se empieza a observar dicha tendencia.

La importancia de los flujos de inversión de portafolio que han arribado a los mercados emergentes siguió la tendencia mundial de ese tipo de inversión y modificó las fuentes tradicionales de financiamiento externo. Ortiz (2004) muestra el cambio en la composición de los flujos privados a los mercados emergentes y la creciente importancia que ha adquirido la inversión de portafolio en dichos flujos. Aunque la inversión de portafolio en los mercados emergentes se ha concentrado principalmente en instrumentos de deuda, preferentemente gubernamental, durante la década pasada también se observó un notable crecimiento de la importancia de los mercados accionarios. Con base en datos sobre los mercados emergentes publicados anteriormente por la Corporación Financiera Internacional y actualmente por la firma privada Standard \& Poor's, Carmichael y Pomerleano (2002) muestran que la capitalización (valor del total de las acciones en circulación a precios de mercado) de 22 de esos mercados en dólares estadounidense alcanzó en la década pasada un crecimiento inusitado, al pasar de 339.3 mil millones en 1990 a 2.2 billones en el año 2000, superando el crecimiento observado en los mercados desarrollados durante ese periodo.

En el caso del mercado mexicano de capitales, el proceso de liberalización resultante de la reforma económica promovida por el gobierno mexicano ha producido un notable incremento en su actividad y en la participación de capitales extranjeros, así como la apertura. ${ }^{2}$ Leriche (1994) considera que las condiciones de la economía interna a partir de 1982 propician un cambio en las políticas de promoción del mercado de valores, pues las autoridades

\footnotetext{
2 Para profundizar en el análisis sobre el proceso de la liberalización financiera en México y sus efectos en la participación del capital extranjero en el mercado de capitales se puede consultar Cabello (1999) y Ortiz (2000).
} 
mexicanas vieron en ese mercado una oportunidad para fomentar el financiamiento de la inversión productiva, así como para lograr la estabilidad económica de largo plazo y el crecimiento sostenido, objetivos importantes y difícilmente alcanzables particularmente porque entre $1983 \mathrm{y}$ 1985, como consecuencia de la restricción financiera impuesta por la crisis de su deuda externa, México no tenía acceso a los créditos en los mercados internacionales. En mayo de 1989 se autorizó el libre acceso de los capitales internacionales al mercado mexicano, salvo en ciertos sectores como el bancario. Ese año llegaron más de 493 millones de dólares al mercado accionario mexicano, iniciándose un periodo de bonanza hasta 1993 en que los flujos externos rebasaron los 10,700 millones de dólares, es decir, en ese periodo el crecimiento observado fue de casi 22 veces. En septiembre de 1989 se emitió el primer ADR mexicano en la bolsa neoyorkina, convirtiéndose ese instrumento en una inversión importante para los extranjeros. Cabello (1999) encuentra que el valor de los ADR en 1989 fue el 49.75\% del valor de mercado de la inversión extranjera en el mercado de capitales mexicano, incrementándose ese porcentaje en los años subsecuentes.

Según datos del Emerging Stock Markets Fact Book (1994-2000), la capitalización del mercado accionario mexicano tuvo un notable crecimiento en el periodo de 1984 a 1999, creció en poco más de 70 veces, mostrando un crecimiento superior al observado por el conjunto de mercados emergentes (que sólo creció poco más de 21 veces). En 1984 la capitalización del mercado mexicano representaba sólo el 1.51\% de la capitalización de los mercados emergentes y en 1999 el 5.01\%, habiendo caído notablemente respecto de 1992 en que fue de $14.03 \%$. Con base en la misma fuente, se puede observar que en dicho periodo la capitalización del conjunto de mercados desarrollados creció casi 10 veces, en tanto que la del mercado mundial lo hizo 10.5 veces. El valor de capitalización del mercado accionario mexicano creció durante el periodo de referencia a una tasa promedio anual de $32.76 \%$, en tanto que la tasa de crecimiento de la capitalización en los mercados emergentes fue de $22.53 \%$; las correspondientes al total de mercados desarrollados y al mercado mundial fueron respectivamente de $16.59 \%$ y $16.95 \%$. Estas cifras muestran el clima de confianza sobre el desempeño del mercado mexicano de acciones, dadas las condiciones existentes durante ese periodo para los inversionistas que según Márquez, Islas y Venegas (2003) se encontraban garantizadas por el programa de ajuste económico (Pacto) y la privatización de empresas paraestatales, incluyendo las más rentables. Sin embargo, en 1994 se redujo notablemente el dinamismo que venía observando el ritmo de ingreso de capitales externos y el flujo durante ese año fue menor a 4,084 
Riesgo sistemático en el mercado mexicano de

capitales: un caso de segmentación parcial

millones de dólares. En particular destaca el hecho de que durante el último trimestre de ese año se observó por primera vez un flujo neto negativo, lo que no ocurría desde 1989, pues las salidas de capital superaron a las entradas por poco más de 373 millones de dólares de acuerdo con datos publicados por Banco de México 3 .

En la sección II se ofrece un resumen de literatura pertinente a este estudio, enfatizando la evidencia que se ha recabado en estudios empíricos previos realizados en torno al riesgo sistemático en México derivado del comportamiento de las variables económicas analizadas en el presente estudio. En la sección III se describe el modelo multifactorial dinámico mediante el cual se analiza la relevancia de los factores de riesgo sistemático de los cuales se ocupa este estudio. La sección IV presenta los resultados del análisis econométrico de la prima de riesgo del mercado mexicano de capitales. Las conclusiones de este estudio se presentan en el último apartado.

\section{Valuación de activos de capital y la integración de los mercados de capitales}

Los principales modelos de valuación de activos son el Capital Asset Pricing Model (CAPM) y el derivado de la Arbitrage Pricing Theory (APT). Con base en los resultados de Markowitz $(1952,1959)$ y las contribuciones de Tobin (1958), trabajando independientemente Treynor $(1961,1962)$, Sharpe $(1963,1964)$, Lintner (1965) y Mossin (1966) desarrollan el primer modelo que ofrece una explicación rigurosa de la fijación de precios en el equilibrio del mercado de capitales como resultado de la exposición de los activos al riesgo sistemático. De acuerdo con dicho modelo teórico sólo existe una fuente de riesgo sistemático: el riesgo de mercado. Por su parte, Ross (1976) propone la APT como alternativa para explicar la fijación de los precios de las activos con base en su grado de exposición al riesgo sistemático. La APT plantea la existencia de diversas fuentes de riesgo sistemático derivadas del comportamiento de variables económicas, no sólo el riesgo de mercado.

En los estudios empíricos basados en modelos de múltiples factores de riesgo sistemático, los factores son extraídos por métodos estadísticos como el análisis factorial o el análisis de componentes principales de la matriz de varianzas-covarianzas (o la de correlaciones) de los rendimientos de un conjunto

\footnotetext{
3 Esta información se publica en el sitio web de Banco de México: www.banxico.org.mx
} 
de acciones, o bien, especificando los factores de riesgo sistemático con base en variables derivadas de la teoría económica (Campbell, Lo y MacKinlay, 1997). Chen, Roll y Ross (1986) representan el riesgo sistemático mediante factores de riesgo derivados de variables macroeconómicas que pueden influir en el precio de los activos por sus efectos en el flujo esperado de dividendos y en los factores de descuento de dichos flujos, encontrando evidencia significativa de la influencia de la tasa de crecimiento de la producción industrial, el diferencial de rendimientos en bonos altamente calificados respecto de bonos de baja calidad crediticia, el diferencial entre los rendimientos de bonos a largo y corto plazo, así como la inflación no esperada. Este estudio de Chen, Roll y Ross ha motivado una amplia agenda de investigación para indagar sobre cuáles son las variables económicas y financieras que producen el riesgo sistemático en diferentes mercados del mundo. ${ }^{4}$ En el caso del mercado de capitales de México existe aún una cantidad escasa de estudios sobre la importancia de las variables económicas para la explicación del riesgo sistemático, entre los que pueden señalarse los efectuados por De la Calle (1990), Nava (1996), Navarro y Santillán (2001) y López y Vázquez (2002) y Al-Shanfari (2003).

Grubel (1968) extendió el alcance de la teoría del portafolio al caso de la inversión internacional, al demostrar que la diversificación de portafolios con la inclusión de activos internacionales permite que el inversionista cuente con mejores oportunidades de reducción del riesgo, pues los rendimientos de los activos de países extranjeros no se encuentran necesariamente altamente correlacionados con los títulos que se negocian en su propio país. Al respecto, Levy y Sarnat (1970) argumentan que en tanto existe una tendencia marcada de que los rendimientos de los diferentes activos de una economía se muevan juntos, es menos probable que los activos de diferentes países observen un comportamiento semejante (Cohn y Pringle, 1973). Lessard (1973) muestra los beneficios que podrían haberse obtenido invirtiendo en activos de Argentina, Brasil, Chile y Colombia concluyendo que, incluso si se diversifican los portafolios en países distintos pero dentro de una misma área geográfica, se pueden obtener importantes ganancias que superan a las obtenidas si la inversión se restringe a activos de un solo país (el estudio de Lessard se refiere al mercado de Estados Unidos).

\footnotetext{
4 Entre los estudios realizados en torno a la importancia de variables económicas y financieras como fuentes de riesgo sistemático en diversos países se pueden citar, entre otros, Boudoukh y Richardson (1993), Chen y Jordan (1993), Clare y Thomas (1994), Dropsy y Nazarim (1995), Schor, Bonomo y Valls (1998), Selamat (2001), Hondroyiannis y Papapetrou (2001), Cagnetti (2002), Altay (2003), Van der Goorbergh, Roon y Werker (2003), Drehman y Manning (2004). Aunque esta lista de estudios representa una ínfima parte de la literatura que se ha producido al respecto, permite tener una idea del interés suscitado.
} 
Riesgo sistemático en el mercado mexicano de

capitales: un caso de segmentación parcial

La inversión de portafolios diversificados internacionalmente también ha motivado el interés académico. Una cuestión medular es cómo se valúan los activos internacionales, surgiendo modelos que extienden el CAPM y el APT al contexto internacional. ${ }^{5} \mathrm{El}$ grado de integración de los mercados adquiere importancia para la valuación de los activos, pues si un mercado está plenamente integrado, los precios de los activos se establecen mediante una base netamente internacional, es decir, el riesgo sistemático mundial es la única fuente pertinente para la valuación. En el extremo opuesto, en un mercado segmentado, los activos se valoran únicamente con base en fuentes de riesgo sistemático locales. Sin embargo, el modelo y los resultados de Errunza y Losq (1985) sugieren que los mercados de capital alrededor del mundo pueden encontrarse en un espectro que va desde la segmentación perfecta hasta la integración perfecta, es decir, existe el caso de segmentación parcial. Una implicación directa de la segmentación parical es que puede haber factores nacionales que determinan el riesgo sistemático y los precios de los activos, junto con la influencia de factores de carácter internacional.

La cuestión de la segmentación vis a vis la integración de los mercados mundiales de capital no es un tema importante únicamente para la literatura sobre la diversificación internacional de portafolios. Además de la importancia de sus efectos en el riesgo y rendimiento de los portafolios desde la perspectiva de los inversionistas, también tiene implicaciones para el financiamiento de las empresas en dichos mercados. Alford (1993) señala que, al contrario que en el caso de los inversionistas de portafolio, las empresas preferirán solicitar fondos en un mercado cuyo grado de segmentación permita que los inversionistas estén dispuestos a pagar un precio más alto por los títulos emitidos que lo que éstas podrían recibir en sus propios países, pues de esta forma se reduciría su costo de capital.

Por otra parte, de acuerdo con los modelos de valuación de activos internacionales, debe haber una reducción en el costo de capital de las empresas de economías segmentadas que pueden tener acceso al mercado internacional de capital, ya que el rendimiento esperado que deben ofrecer esas empresas a los inversionistas internacionales se reduce debido a que se les ofrece un mayor número de oportunidades para disminuir el riesgo de sus portafolios mediante la diversificación. Errunza y Miller (2000) estudian los cambios en los costos de capital de 126 empresas de 32 países, antes y después de la liberalización de esos

5 Alford (1993) presenta un buen análisis al respecto. 
mercados, encontrando que el costo de capital se redujo en $42 \%$, resultado congruente tanto con lo que sugieren al respecto los modelos de valuación de activos internacionales, como con los resultados de otros estudios, como el de Henry (2000) y el de Bekaert y Harvey (2000).

Este documento hace una contribución doble al avance en la comprensión del riesgo sistemático en el mercado mexicano de capitales mediante la identificación de la importancia que tienen variables macroeconómicas clave. El conjunto de las variables económicas analizadas incluye tanto variables domésticas como internacionales, por lo que el análisis empírico permite obtener evidencia sobre la hipótesis conjunta de que son relevantes las variables económicas analizadas para explicar el riesgo sistemático en el mercado mexicano de capitales y de que este mercado aún está parcialmente segmentado del mercado mundial de capitales.

\section{Modelo multifactorial del riesgo sistemático en el mercado mexicano de capitales}

El modelo dinámico para llevar a cabo el análisis subsecuente puede representarse de manera general como:

$$
r_{t}=\alpha_{0}+\sum_{i=1}^{p} \alpha_{i} r_{t-i}+\sum_{i=1}^{k} \sum_{j=0}^{l} \beta_{i, j} X_{i, t-j}+\psi^{\prime} \delta+\varepsilon_{t}, \quad t=1,2, \ldots, T
$$

donde $r_{t}$ es el rendimiento del mercado en exceso de la tasa libre de riesgo, es decir, la prima por riesgo del mercado, ${ }^{6} X_{t}$ son observaciones de las variables explicativas, los factores de riesgo representados por las componentes principales obtenidas por López y Ortiz (2005) menos la tasa libre de riesgo; ${ }^{7}$ por lo que se pueden considerar como las primas correspondientes al factor de riesgo. $\delta$ es una matriz con variables ficticias (dummies), cuyo objetivo es recoger los efectos que pudieran tener eventos cuya naturaleza puede alterar la distribución probabilística

6 En lo sucesivo se denomina MXSMPR a la prima de riesgo del mercado mexicano de capitales.

7 En lo sucesivo se hará referencia a los factores de riesgo como MXCP1RP, MXCP2RP..., para designar a las primas de riesgo de los factores de riesgo sistemático. 
Riesgo sistemático en el mercado mexicano de

capitales: un caso de segmentación parcial

de la prima de riesgo. Por su parte, las $a$, las by el vector $y$ 'son parámetros a estimar que, bajo los supuestos de este estudio, muestran la relación entre los factores de riesgo y la prima de riesgo en cada mercado; de manera específica, los parámetros muestran cuál es la recompensa agregada del mercado por su exposición al riesgo sistemático. Finalmente, $\varepsilon_{\tau}$ es un término de perturbación aleatoria contemporánea. Se incluyen variables rezagadas en el modelo, pues los inversionistas toman decisiones con base en el conjunto de información disponible, formando los eventos pasados parte de ese conjunto, y el comportamiento de las variables económicas puede surtir efectos durante varios periodos posteriores, pudiendo ser el caso que incluso los efectos no se presenten contemporáneamente, sino que empiecen a resentirse hasta después de que ha transcurrido cierto tiempo.

Navarro y Santillán (2001), Márquez, Islas y Venegas (2003) y Al-Shanfari (2003) muestran evidencia de que la tasa de interés es un factor de riesgo importante en el mercado accionario mexicano. En los estudios de De la Calle (1990), Nava (1996), Navarro y Santillán (2001) y de Al-Shanfari (2003) se ofrece evidencia de la influencia de la oferta monetaria en el caso del mercado accionario mexicano. Lothian y McCarthy (2001), en un estudio sobre 14 países desarrollados, encuentran que las acciones son una cobertura contra la inflación sólo a muy largo plazo, pues los precios accionarios se ajustan a la inflación pero sólo después de un plazo de tiempo prolongado. Este resultado es consistente con el hallazgo de Cagan (1974), según el cual se observa un periodo prolongado, mayor a una década, en el ajuste de los mercados accionarios a la inflación. Para el caso de México, De la Calle (1990), Nava (1996) y Navarro y Santillán (2001) han encontrado evidencia significativa del efecto de la inflación; asimismo López y Vázquez (2002) muestran que los cambios en el nivel de precios contribuyen a explicar los rendimientos de varias acciones mexicanas. Navarro y Santillán (2001) encuentran que las exportaciones son significativas para explicar los rendimientos en el mercado accionario mexicano (las exportaciones contribuyen a generar empleo, lo que incide favorablemente en el ingreso, el consumo y en la producción). Por otra parte, Bailey y Chung (1986), así como Nava (1996) y Navarro y Santillán (2001), encuentran evidencia de la importancia del riesgo cambiario en el mercado accionario mexicano. Aunque en la literatura empírica previa sobre el riesgo sistemático no se ha estudiado, López y Ortiz (2005) incluyen en su análisis el comportamiento de las exportaciones mundiales, pues consideran que éstas dan cuenta de la actividad económica en el nivel mundial, debiendo estar significativamente relacionadas con los mercados accionarios debido a que un mayor nivel de exportaciones mundiales implica mayores niveles 
de ingreso, empleo y producción en el mundo, y, por tanto, su comportamiento puede indicar el estado de confianza respecto del desempeño económico. De las importaciones se espera el efecto contrario.

En la literatura previa sobre el riesgo sistemático en México no se ha analizado el efecto que pudiesen tener las importaciones, sin embargo, Cagnetti (2002) encuentra que las importaciones son relevantes para explicar el riesgo sistemático en el mercado accionario italiano. Aunque en primera instancia se puede esperar que las importaciones muestren un efecto contrario al de las exportaciones, por representar la parte de la demanda agregada que no es satisfecha por las empresas domésticas, es importante considerar que las importaciones de maquinaria y equipo pueden obedecer a que existe confianza en las condiciones presentes y/o futuras para la inversión empresarial en tanto que el componente de las importaciones correspondiente al consumo de las familias podría indicar que ha aumentado el ingreso o que existen expectativas de que se incremente en el corto plazo.

En diversos estudios del riesgo sistemático y de la valuación de activos mexicanos se incluyen variables internacionales para capturar los efectos de factores de riesgos internacionales. Por ejemplo, De la Calle (1990) encuentra que los cambios en el índice accionario estadounidense Standard \& Poors 500 son un factor de riesgo para las acciones mexicanas, en tanto que los resultados de Nava (1996) muestran que el índice Dow Jones es un factor de riesgo. Por su parte, Navarro y Santillán (2001) incluyen en su estudio del mercado mexicano al índice Dow Jones y la tasa de los Certificados de la Tesorería, Treasury bills, de Estados Unidos para depósitos a plazos mayores a tres meses encontrando una relación significativa en ambos casos. Nava (1996) observa una relación significativa entre la producción industrial estadounidense y algunas acciones mexicanas. Aunque la investigación de Galindo y Guerrero (1999) no tiene como objetivo el análisis del riesgo sistemático mexicano, es de señalarse que muestran evidencia de una relación de largo plazo significativa entre el índice Dow Jones y el principal indicador bursátil del mercado accionario mexicano, el IPC. Estos indicadores internacionales no dan cuenta de la integración del mercado mexicano de capitales al mercado mundial, pero Al-Shanfari (2003) encuentra que el índice mundial de precios accionarios FT-S\&P es un factor de riesgo importante para el mercado mexicano. 
Riesgo sistemático en el mercado mexicano de

capitales: un caso de segmentación parcial

En el cuadro 1, se presentan las variables analizadas por López y Ortiz (2005) y de cuya matriz de correlaciones obtienen las componentes principales. Todas las variables se expresan en dólares estadounidenses y sus tasas de crecimiento se construyen con índices que tienen su periodo base en diciembre de 1983. En el caso de las tasas de interés, también en términos de esa divisa, se consideran cambios en lugar de tasas de crecimiento.

\section{Cuadro 1}

Variables económicas que forman los componentes principales

Variable

Descripción

MXUSERR Tasa de depreciación del peso mexicano frente al dólar estadounidense

MXIPG Tasa de crecimiento de la producción industrial en México

MXCPIG Tasa de crecimiento de la inflación en México

MXMOG Tasa de crecimiento de la oferta monetaria en México

MXXG Tasa de crecimiento de las exportaciones mexicanas

MXMG Tasa de crecimiento de la importaciones mexicanas

MXIC Cambio en la tasa del mercado monetario en México

MXTBILLC Cambio en la tasa de rendimiento del Cete a 28 días

WXG Tasa de crecimiento de las exportaciones mundiales

Fuente: López y Ortiz (2005)

En el cuadro 2 se reproducen los valores propios que muestran la cantidad de la varianza total del conjunto de variables originales que explica cada componente principal. En el cuadro 3 se presentan los vectores de carga de las tres primeras componentes principales, mostrando el grado de asociación lineal (correlación) existente entre las variables analizadas y las componentes principales. ${ }^{8}$

\footnotetext{
8 López y Ortiz (2005) prosiguen su análisis únicamente con las tres primeras componentes. Justifican su decisión con base en el criterio del valor propio para la selección de las componentes principales a retener para análisis posteriores y en su posible interpretación económica.
} 


\section{Cuadro 2}

Valores propios y porcentaje de varianza explicada por cada una de las componentes principales

\begin{tabular}{llll}
\hline Componente & $\begin{array}{l}\text { Valor } \\
\text { propio }\end{array}$ & $\begin{array}{l}\text { Varianza } \\
\text { explicada\% }\end{array}$ & $\begin{array}{l}\text { \%Varianza } \\
\text { acumulada }\end{array}$ \\
\hline 1 & $\mathbf{3 . 0 6 8}$ & 34.087 & 34.087 \\
2 & $\mathbf{2 . 4 2 2}$ & 26.913 & 60.999 \\
3 & $\mathbf{1 . 6 9 6}$ & 18.806 & 79.805 \\
4 & 0.802 & 8.906 & 88.711 \\
5 & 0.410 & 4.555 & 93.266 \\
6 & 0.246 & 2.730 & 95.996 \\
7 & 0.212 & 2.361 & 98.356 \\
8 & 0.0824 & 0.915 & 99.272 \\
9 & 0.066 & 0.728 & 100 \\
\hline
\end{tabular}

Fuente: López y Ortiz (2005)

Cuadro 3

Vectores de cargas

\begin{tabular}{lccc}
\hline \multicolumn{3}{c}{ Componente } & \\
\hline Variable & 1 & 2 & 3 \\
MXUSERR & $\mathbf{- 0 . 9 1 0 3 0 7 7 8}$ & 0.18623646 & 0.24321104 \\
MXIPG & $\mathbf{0 . 8 4 5 3 1 9 4 3}$ & 0.32141180 & -0.06770787 \\
MXCPIG & $\mathbf{0 . 8 1 9 2 5 8 7 1}$ & -0.18789713 & -0.39032203 \\
MXMOG & $\mathbf{0 . 6 5 3 8 2 4 0 3}$ & -0.04703816 & -0.24070586 \\
MXXG & 0.18771164 & $\mathbf{0 . 8 4 7 0 6 8 9 7}$ & 0.20508716 \\
MXMG & 0.24247777 & $\mathbf{0 . 8 3 8 7 4 4 2 9}$ & 0.24055425 \\
WXG & 0.07861069 & $\mathbf{0 . 6 9 3 8 5 0 8 5}$ & 0.25028775 \\
MXIC & 0.39173306 & -0.42323903 & $\mathbf{0 . 7 9 3 1 8 8 4 8}$ \\
MXTBILLC & 0.41501050 & -0.40625723 & $\mathbf{0 . 7 9 1 7 0 5 1 0}$ \\
MXUSERR $=$ tasa de depreciación del peso frente al USD & \\
MXIPG $=$ tasa de crecimiento de la producción industrial & \\
MXCPIG $=$ tasa de crecimiento del nivel de precios & \\
MXMOG $=$ tasa de crecimiento de la oferta monetaria & \\
MXXG $=$ tasa de crecimiento de las exportaciones mexicanas & \\
MXMG $=$ tasa de crecimiento de las importaciones mexicanas & \\
WXG $=$ tasa de crecimiento de las exportaciones mundiales & \\
MXIC $=$ cambios en la tasa de interés del mercado de dinero & \\
MXTBILLC $=$ cambios en el CETE & \\
\hline
\end{tabular}

Fuente: López y Ortiz (2005) 
Riesgo sistemático en el mercado mexicano de

capitales: un caso de segmentación parcial

Los vectores de carga facilitan la interpretación de las relaciones existentes entre las variables que se analizan. Las variables más asociadas con la primera componente son la tasa de cambio en la cotización del dólar y las tasas de crecimiento de la producción industrial, la inflación y la oferta monetaria, por lo que dicha componente parece describir las condiciones del desempeño de la economía real. El signo negativo de la primera correlación y positivo de las otras sugiere un proceso en el cual a menor tasa de depreciación del peso mexicano ante la moneda estadounidense se incentiva la actividad industrial al mismo tiempo que crece la oferta monetaria y el nivel de precios. El aumento de la oferta monetaria puede explicarse como una respuesta a las necesidades de liquidez por el incremento en el consumo privado y la demanda de inversión que se produce al aumentar la actividad industrial. La mayor demanda agregada y la mayor liquidez existente pueden ejercer, como es sabido, presiones inflacionarias y, en ese contexto, se puede explicar la correlación positiva entre la misma componente y los cambios en la tasa de interés del mercado de dinero, ya que esas asociaciones pueden obedecer a las presiones al alza de dicha tasa debido tanto al aumento de la demanda de créditos de corto plazo y a las expectativas sobre la inflación originadas por el aumento en el nivel de precios que hace aumentar la prima para compensar el riesgo de la pérdida del poder adquisitivo.

Las variables con mayor importancia para explicar la segunda componente son las tasas de crecimiento de las exportaciones mundiales y de las domésticas, así como la de las importaciones, todas ellas con signo positivo, por lo que se infiere que esta componente muestra la influencia de los efectos derivados del estado de la economía mundial en la economía mexicana (dicha componente explica casi el $27 \%$ de la variación total del conjunto de variables, véase cuadro 2). Aunque con una carga baja, la correlación positiva de la tasa de crecimiento de la producción industrial con esta componente principal muestra la obvia vinculación entre el crecimiento del comercio mundial y la actividad industrial doméstica. El tercer vector de cargas muestra que las variables más correlacionadas con la componente 3 son las tasas de interés del mercado monetario y la tasa de los Cetes con asociación positiva, por lo que dicha componente pudiera representar las condiciones del mercado de dinero. 


\section{Análisis econométrico}

En el cuadro 4 se presentan los resultados de las pruebas de raíces unitarias de las variables dependiente e independientes del modelo presentado en la ecuación (1) de la sección anterior, con objeto de verificar si las series son estacionarias o no. ${ }^{9}$ Tanto la prueba de Dickey y Fuller, como la de Phillips y Perron, permiten concluir que las series de interés pueden tratarse como estacionarias para efectos de la estimación correspondiente al modelo econométrico representado por la ecuación (1). Una vez que se verificó la estacionariedad de las series, se procedió a estimar los parámetros de la ecuación (1).

\section{Cuadro 4 \\ Pruebas de raíces unitarias de las primas de riesgo y de las variables explicativas}

\begin{tabular}{ccccccc}
\hline Variable & \multicolumn{3}{c}{ Prueba de Dickey $y$ Fuller } & \multicolumn{3}{c}{ Prueba de Phillips y Perron } \\
\cline { 2 - 7 } & $\hat{\tau}_{\beta}$ & $\hat{\tau}_{\mu}$ & $\hat{\tau}$ & $\tilde{t}_{\beta}$ & $\tilde{t}_{\mu}$ & $\tilde{t}$ \\
MXSMRP & -11.8859 & -11.90629 & -11.88975 & -11.58902 & -11.62544 & -11.62727 \\
& $(<0.01)$ & $(<0.01)$ & $(<0.01)$ & $(<0.01)$ & $(<0.01)$ & $(<0.01)$ \\
MXCP1RP & -4.715812 & -4.004314 & -3.983363 & -12.16695 & -9.984977 & -9.623234 \\
& $(<0.01)$ & $(<0.01)$ & $(<0.01)$ & $(<0.01)$ & $(<0.01)$ & $(<0.01)$ \\
MXCP2RP & -4.105369 & -4.989822 & -5.193123 & -17.17264 & -16.67433 & -16.01074 \\
& $(<0.01)$ & $(<0.01)$ & $(<0.01)$ & $(<0.01)$ & $(<0.01)$ & $(<0.01)$ \\
MXCP3RP & -6.332118 & -3.777054 & -4.039627 & -9.002729 & -7.009106 & -6.60967 \\
& $(<0.01)$ & $(<0.01)$ & $(<0.01)$ & $(<0.01)$ & $(<0.01)$ & $(<0.01)$
\end{tabular}

Para controlar los efectos de la autocorrelación, en la prueba de Dickey y Fuller se efectúa la selección de rezagos con el criterio de información de Schwarz a partir de una especificación inicial de 14 rezagos, en el caso de la prueba de Phillips y Perron se escoge la banda de Newey y West.

$\hat{\tau}_{\beta}$ y $\quad \tilde{t}_{\beta} \quad$ corresponden a la prueba con intercepto y tendencia lineal: $\Delta x \tau=\mu+\gamma x_{\tau-1}+\beta_{\tau}+\eta_{\tau}$ $\mathrm{H}_{0}: x_{\tau}=x_{\tau-1}+\eta_{\tau}, \mathrm{H}_{\mathrm{a}}: x_{\tau}=\mu+\phi x_{\tau-1}+\beta \tau+\eta_{\tau}, \phi<1, \phi-1=\gamma$

$\hat{\tau}_{\mu}^{0} \mathrm{y}{ }^{\tau} \tilde{t}_{\mu}$ corresponden a la prueba con intercepto y sin tendencia lineal: $\Delta x_{\tau}=\mu+\gamma x_{\tau-1}+\eta_{\tau}$ $H_{0}: x_{\tau}=x_{\tau-1}+\eta_{\tau}, H_{\mathrm{a}}: x_{\tau}=\mu+\phi x_{\tau-1}+\eta_{\tau}, \phi<1, \phi-1=\gamma$

$\hat{\tau}$ y $\tilde{t}$ corresponden a la prueba sin intercepto y sin tendencia lineal: $\Delta x_{\tau}=\gamma x_{\tau-1}+\eta_{\tau}$ $H_{0}: x_{\tau}=x_{\tau-1}+\eta_{\tau}, H_{\mathrm{a}}: x \tau=\phi x_{\tau-1}+\eta_{\tau}, \phi<1, \phi-1=\gamma$

Los números entre paréntesis son los valores $\mathrm{p}$, propuestos por MacKinnon, que denotan el nivel de significatividad marginal alcanzado en la prueba

9 Es de interés verificar que todas las series, tanto las de la variable dependiente como las de las explicativas, sean estacionarias, pues de ser así permiten estimar ecuaciones equilibradas en el orden de integración, lo que a su vez permite validar la estimación del modelo mediante pruebas de inferencia estadística realizadas conforme a las distribuciones de probabilidades convencionalmente utilizadas (Maddala y Kim, 2000). 
Riesgo sistemático en el mercado mexicano de

capitales: un caso de segmentación parcial

Se incluyó en la especificación del modelo econométrico la prima de riesgo del mercado mundial de capitales, representada por el exceso de los rendimientos mensuales del portafolio del mercado mundial de capitales ${ }^{10}$ sobre la tasa Libor para eurodepósitos a un mes, para dar cuenta de la integración del mercado mexicano con el mercado mundial. ${ }^{11} \mathrm{Se}$ incluyeron variables tipo dummy, con valor de uno en el periodo en que ocurrieron eventos específicos y cero en cualquier otro periodo, como el crack de octubre de 1987 (crack87) y diversas crisis como la mexicana de 1994 (tequila), la asiática de 1997 (dragon), la rusa de 1998 (vodka) y la brasileña de 1999 (real). Otras variables dummies que se consideraron fueron la denominada NAFTA, con valores de uno a partir de enero de 1994 y de cero para todos los meses previos con el objetivo de verificar si el TLCAN ha tenido efectos en las prima de riesgo del mercado accionario mexicano. También se incluyó la variable estabilización con valor de cero hasta noviembre de 1987 y uno para el resto de las observaciones. Se utilizaron alternativamente dos variables tipo dummy para recoger los efectos de la liberalización del mercado mexicano de capitales: una con valor de cero hasta abril de 1989 y de uno después de ese periodo y otra con valor de cero hasta agosto de 1989 y con valor de uno a partir de septiembre de ese año. ${ }^{12}$

Mediante un proceso de reducción secuencial se descartaron las variables explicativas que no alcanzaron cuando menos un nivel de $10 \%$ de significatividad marginal (probabilidad de cometer el error de Tipo I), obteniéndose finalmente el modelo cuya estimación se muestra en el cuadro 5, mismo que incluye los rezagos significativos de la prima de riesgo del mercado mexicano y de los factores de riesgo sistemático. ${ }^{13}$ Los coeficientes estimados de las variables son altamente

${ }^{10}$ Obtenidos del índice mundial de mercados de capitales de Morgan Stanley Capital Internacional (MSCI).

11 Tanto en Carrieri, Errunza y Hogan (2001) como en Arouri (2004), se utiliza también la tasa para eurodepósitos a un mes para representar la tasa mundial libre de riesgo.

12 En mayo de 1989 las autoridades gubernamentales mexicanas anunciaron la liberalización del mercado mexicano de valores y en septiembre del mismo año empezó a cotizar en la Bolsa de Valores de Nueva York (NYSE) el primer ADR mexicano.

${ }^{13}$ El proceso de reducción se inicia estimando una especificación del modelo tan grande como sea posible. Los parámetros no significativos se someten a una prueba de restricciones, verificando que en el modelo restringido (reducido) los criterios de evaluación sean mejores que el modelo sin restricciones. El proceso continúa hasta el punto en que es posible reparametrizar el modelo o no existen parámetros no significativos, obteniendo así un modelo lo más parsimonioso posible y congruente en términos de la teoría económica. Este procedimiento es congruente con el enfoque de modelación de lo general a lo específico. 
significativos, exceptuando el correspondiente al décimo rezago de MXCP3RP, el cual lo es en un nivel marginalmente superior al $5 \%$. Según la $\mathrm{R}^{2}$, en conjunto, las variables dentro del modelo explican poco más del $60 \%$ de las variaciones de la prima de riesgo en el mercado accionario mexicano. El valor alcanzado por el estadístico de Jarque y Bera no permite rechazar la hipótesis de normalidad de los residuales de la ecuación estimada. No se encontró evidencia de heteroscedasticidad y la prueba Reset de Ramsey no muestra evidencia de que existan problemas de especificación. Sin embargo, al realizar la prueba pormanteu de Ljung y Box a los residuales, se detectaron autocorrelaciones positivas en los rezagos dos y cinco, aunque sólo son significativas al 10\%. También se detectó la presencia de un efecto ARCH significativo, corroborándose la presencia de dicho efecto mediante la prueba pormanteu de los cuadrados de los residuos de la ecuación.

De las variables utilizadas para representar a los factores de riesgo sistemático la más significativa es la correspondiente a MXCP1RP, con un coeficiente superior a 3.3331, también es significativo su rezago séptimo, aunque sólo se puede considerar significativo al $5 \%$ y con un coeficiente de menor magnitud (ligeramente superior a 0.699). Los rezagos quinto, séptimo y décimo de la variable MXCP3RP también son significativos, resultando que los valores de los coeficientes estimados son de $1.386047,1.583049$ y 0.851159 , respectivamente, siendo el último el de menor magnitud y sólo significativo al $10 \%$. La no significatividad del riesgo asociado con el comportamiento del comercio internacional mexicano y mundial, capturado mediante la variable MXCP2RP, puede deberse a que sus posibles efectos han sido capturados en los otros coeficientes que resultan significativos en la ecuación.

El coeficiente asociado con el valor contemporáneo de MXCP1RP, además de señalar que la exposición del mercado a dicho factor es la más importante, dado el signo positivo con que aparece en la ecuación estimada, sugiere que los rendimientos del mercado se elevan en la medida en que crece la producción industrial y existe un entorno con estabilidad cambiaria. Dado que el factor de riesgo construido con la primera componente principal captura los efectos que tiene la inflación en el comportamiento de la actividad económica, se desprende de la estimación del modelo que los rendimientos en el mercado accionario pueden ser una cobertura para la inflación, pues recompensan al inversionista por el riesgo de la pérdida del poder adquisitivo. Asimismo, la incertidumbre cambiaria, capturada en esa misma componente por la tasa de depreciación del peso respecto del dólar de los Estados Unidos, reduce la recompensa por el riesgo. Esto puede 
Riesgo sistemático en el mercado mexicano de capitales: un caso de segmentación parcial

\section{Cuadro 5 \\ Modelo de la prima de riesgo estimado para el mercado mexicano de capitales}

$$
r_{t}=\alpha_{0}+\sum_{i=1}^{p} \alpha_{i} r_{t-1}+\sum_{i=1}^{k} \sum_{j=0}^{l} \beta_{i, l} X_{i, t-j}+\psi^{\prime} \delta+\varepsilon_{t}
$$

Variable dependiente: rendimiento del mercado en exceso de la tasa libre de riesgo

\begin{tabular}{|c|c|c|c|c|}
\hline Variable independiente & Coeficiente & Error estándar & $t$ & Valor- $p$ \\
\hline Intercepto & 29.79453 & 3.467057 & 8.593608 & $<0.01$ \\
\hline MXCP1RP & 3.333101 & 0.339130 & 9.828400 & $<0.01$ \\
\hline $\mathrm{MXCP} \mathrm{RP}_{\mathrm{t}-7}$ & 0.699068 & 0.347269 & 2.013043 & 0.0454 \\
\hline $\mathrm{MXCP} \mathrm{RP}_{\mathrm{t}-5}$ & 1.386047 & 0.483189 & 2.868537 & $<0.01$ \\
\hline $\mathrm{MXCP} \mathrm{RP}_{\mathrm{t}-7}$ & 1.583049 & 0.489634 & 3.233125 & $<0.01$ \\
\hline $\mathrm{MXCP} 3 \mathrm{RP}_{\mathrm{t}-10}$ & 0.851159 & 0.436956 & 1.947926 & 0.0528 \\
\hline CRACK87 & -73.56751 & 6.506447 & -11.30686 & $<0.01$ \\
\hline ESTABILIZACION & -22.37618 & 3.407775 & -6.566213 & $<0.01$ \\
\hline ADR & -6.170827 & 2.288154 & -2.696858 & $<0.01$ \\
\hline NAFTA & -3.097350 & 1.420947 & -2.179778 & 0.0304 \\
\hline VODKA & -22.96762 & 8.701067 & -2.639632 & $<0.01$ \\
\hline WCMPRP & 0.688886 & 0.138953 & 4.957704 & $<0.01$ \\
\hline \multicolumn{5}{|l|}{ Medidas de evaluación estadística: } \\
\hline R cuadrada & 0.604155 & & & \\
\hline R cuadrada ajustada & 0.583018 & & & \\
\hline$\chi_{\text {normalidad (Jarque y Bera) }}^{2}$ & 1.313496 & & & 0.518135 \\
\hline$F_{\text {autocorrelación }}(12$ rezagos) & 1.072840 & & & 0.385041 \\
\hline $\begin{array}{l}\chi_{\text {autocorrelación (L-M Breusch y Godfrey), }} \\
12 \text { rezagos }\end{array}$ & 13.56646 & & & 0.329245 \\
\hline$F_{\text {heteroscedasticidad }}$ & 1.175003 & & & 0.287627 \\
\hline$\chi_{\text {heteroscedasticidad }}^{2}$ & 19.79570 & & & 0.284812 \\
\hline Arch-12 & 25.70875 & & & 0.011799 \\
\hline$F_{\text {RESET }}$ & 0.174280 & & & 0.676773 \\
\hline
\end{tabular}

explicarse porque ante la incertidumbre con respecto al tipo de cambio, los inversionistas pueden preferir modificar las tenencias de sus portafolios, vendiendo títulos denominados en moneda mexicana para adquirir activos denominados en dólares como medio de protección ante posibles pérdidas derivadas de la depreciación futura del peso ante el dólar y de las pérdidas que podrían darse como consecuencia de cambios en el desempeño de las empresas inducidos por el comportamiento de la paridad cambiaria. 
Los rezagos significativos de MXCP3RP, cuyo efecto conjunto es de magnitud equiparable al de la variable MXCP1RP, indican que las alzas pasadas en las tasas de interés tienen efectos en la prima de riesgo contemporánea. El signo positivo de los coeficientes correspondientes implica que la reducción que se da los precios de los activos los hace más atractivos a los inversionistas. En el transcurso del tiempo la demanda de títulos eleva sus precios, haciendo que el efecto posterior al alza de las tasas sea un aumento en los rendimientos del mercado accionario. Por otra parte, los signos positivos en los coeficientes de los valores rezagados de MXCP3RP confirman que los rendimientos de títulos accionarios son una cobertura contra la inflación, pues las variables con mayor carga vectorial (véase cuadro 3) dentro de esa componente principal son los cambios en las tasas de interés, los cuales están relacionados por la teoría económica con las expectativas de aumentos en el nivel de precios. Estos resultados son similares a los obtenidos en las investigación de Hondroyiannis y Papapetrou (2001) y la de Dritsaki-Bargiota y Dritsaki (2004), quienes encuentran que en el caso del mercado de capitales de Atenas la tasa de inflación pasada y los cambios rezagados de las tasas de interés causan en el sentido de Granger a los rendimientos del mercado.

De acuerdo con la estimación, el coeficiente para capturar la relación entre la prima de riesgo y el periodo en que han estado vigentes las políticas de estabilización económica muestra que existen efectos significativos en el mercado de capitales, indicando una reducción en la prima de riesgo del mercado. En los resultados de la estimación, también se observa que la entrada en vigor del TLCAN tuvo un efecto significativo, evidenciando una reducción en el nivel de la prima de riesgo de casi $3.1 \%$. La variable asociada con la emisión del primer ADR mexicano es significativa, lo que indica una reducción en el nivel de la prima de riesgo de poco más del 6\%. Cuando en la especificación del modelo se incluyó como alternativa la variable dummy que considera el efecto de la fecha del decreto oficial de liberalización no mostró significatividad alguna. Este resultado puede sugerir que en la dinámica del mercado es más importante el hecho de que los inversionistas, internacionales tengan acceso efectivo al mercado de capitales, que el anuncio oficial del retiro de barreras existentes para la inversión extranjera. Los ADR resultan atractivos a los inversionistas, pues les permiten disfrutar de la propiedad accionaria de una empresa extranjera sin tener que enfrentarse a problemas cambiarios, fiscales, de custodia y liquidación. El coeficiente que asocia la prima de riesgo del mercado accionario mexicano con la prima de riesgo del mercado mundial de capitales es positiva y altamente significativa, con una magnitud de 6.89 
Riesgo sistemático en el mercado mexicano de

capitales: un caso de segmentación parcial

aproximadamente. Este resultado evidencia que la prima de riesgo del mercado mexicano está relacionada positivamente con la prima de riesgo del mercado mundial de capitales.

\section{Conclusiones}

La exposición de la prima de riesgo del mercado mexicano de capitales al factor del riesgo, con el cual se encuentra fuertemente asociada la incertidumbre cambiaria, muestra que puede haber efectos de importancia en el mercado de activos como consecuencia de la transmisión de movimientos bruscos en el tipo de cambio. Estos resultados explican porque en los estudios de Bailey y Chung (1995), Nava (1996), Navarro y Santillán (2001), Doshi, Johnson, Ortiz y Soenen (2001) y de Navarro y Santillán (2001), el tipo de cambio es una variable importante para explicar el comportamiento general del mercado accionario mexicano.

Por otra parte, se evidencia también que el crecimiento de la oferta monetaria incrementa los precios de los títulos, lo que puede explicarse como consecuencia de la demanda de títulos derivada de la expansión de la liquidez tal como lo proponen la literatura teórica y los resultados de la investigación empírica. Por lo tanto, los resultados de esta investigación respecto a la importancia del crecimiento de la oferta monetaria en la prima de riesgo del mercado mexicano de capitales son congruentes con los resultados de estudios previos sobre este mercado, tales como los presentados por De la Calle (1990), Nava (1996), Navarro y Santillán (2001) y en Al-Shanfari (2003).

De acuerdo con las estimaciones realizadas, este estudio corrobora que los rendimientos en el mercado accionario mexicano son una cobertura para la inflación como lo demuestran el estudio de Doshi, Johnson, Ortiz y Soenen (2001) y el de Cabello, De Jesús y Ortiz (2004) explicando de esta forma porque los rendimientos de los activos reflejan los efectos de los cambios en el nivel de precios, como se observa en la investigación de López y Vázquez (2002).

El efecto significativo mostrado por la variable estabilización en este estudio es congruente con el hallazgo del estudio efectuado por De la Calle (1990) sobre la existencia de primas en exceso debido a la inestabilidad de las condiciones macroeconómicas en México. La existencia de una menor prima de riesgo para el periodo previo al proceso de estabilización no se puede explicar por un menor nivel de 
riesgo sistemático, sino porque, a pesar de la tendencia alcista del indicador del mercado bursátil mexicano, existieron altas tasas de interés motivadas a su vez por las altas tasas de inflación que se venían observando en la economía mexicana, a diferencia del periodo posterior, en el cual las tasas de interés han descendido notoriamente. Además de proporcionar a los inversionistas una mejor recompensa por el riesgo durante el segundo subperiodo, se observa un descenso importante en la desviación estándar de la prima de riesgo, de lo que se puede concluir que este subperiodo se caracteriza por un menor nivel general de riesgo. En resumen, se puede afirmar que en el periodo de estabilización se observa una prima de riesgo más alta en promedio y con mayor estabilidad.

La significatividad de la variable que recoge los efectos del TLCAN sugiere la posibilidad de mayor integración del mercado mexicano de capitales con respecto a los mercados de capitales de Canadá y Estados Unidos. Este resultado es congruente con el de Darrat y Zhong (2001), quienes no encuentran evidencia de integración para el periodo previo a la vigencia del TLCAN, pero sí la encuentran una vez que éste entró en vigor.

El que la fecha del anuncio oficial de liberalización del mercado accionario mexicano no haya resultado significativo no quiere decir necesariamente que carezca de importancia; de hecho, se puede esperar que entre la publicación del anuncio y la instrumentación de los arreglos institucionales y operativos para ponerlo en marcha transcurra cierto tiempo. Debe tomarse en cuenta que la emisión del primer ADR mexicano en la Bolsa de Nueva York se pudo llevar a cabo gracias a que las autoridades establecieron el marco normativo que lo permitió. Es el caso, entonces, que los efectos del anuncio de la liberalización del mercado accionario mexicano se manifestaron con posterioridad y precisamente a través de la emisión del ADR. Por otra parte, como señalan Bekaert, Harvey y Lundblad (2003), los efectos de un proceso de liberalización pueden no presentarse si existen otras deficiencias en el mercado que se pretende liberalizar. Un anuncio oficial per se no elimina otras deficiencias presentes en el mercado, como pudieran ser algunos de los problemas relacionados con las características de la información sobre las empresas y la marcha de la economía, por lo que en el caso del mercado mexicano de acciones la presencia de problemas de información asimétrica pudiera haber inhibido la participación inmediata de los inversionistas extranjeros, impidiendo que se manifestasen de inmediato los efectos del anuncio en el mercado. 
Riesgo sistemático en el mercado mexicano de

capitales: un caso de segmentación parcial

Aunque Bekaert, Harvey y Lundblad mencionan también que la falta de credibilidad en el anuncio oficial de apertura del mercado pudiera ser un obstáculo para el proceso de liberalización, éste no parece ser el caso pues en junio de 1989, el mes siguiente al del anuncio, se emitió el primer bono internacional mexicano después de la crisis de la deuda de 1982. Cabe destacar que los resultados de este estudio son congruentes con los resultados de Bekaert y Harvey (2000), Henry (2000), Errunza y Miller (2000) y Fernandes (2002), quienes han encontrado evidencia sobre la reducción de la prima de riesgo para las empresas que emiten un ADR, mostrando además que puede tener efectos semejantes sobre otros activos que se negocian en el mercado del país de la empresa emisora.

El hecho de que los coeficientes de algunas variables rezagadas sean significativos para explicar la prima de riesgo del mercado accionario mexicano, puede interpretarse como evidencia de que la información económica pasada es importante para los inversionistas. Los rezagos significativos muestran los efectos que tienen las expectativas que se forman los inversionistas con base en la información económica disponible para la toma de decisiones de inversión en activos riesgosos mexicanos. Por la misma razón, se pueden explicar las autocorrelaciones débilmente significativas que exhibe la serie de residuales de la ecuación estimada. Es decir, en el conjunto de información que usan los inversionistas para la toma de decisiones se incorpora una parte importante de la información pasada. En particular, debe considerarse que una parte muy importante de la información únicamente está disponible en forma trimestral y, en general, toda la información económica se publica con algún retraso, de ahí que en las expectativas de los inversionistas tenga peso su conocimiento sobre el comportamiento pasado de las variables económicas.

La evidencia con respecto a la importancia de la prima de riesgo del mercado mundial de capitales sugiere que el mercado mexicano está parcialmente segmentado porque, además de que el riesgo sistemático del mercado mundial se refleja en la prima de riesgo del mercado local, otros factores de riesgo sistemático de tipo doméstico ejercen también influencia en esta prima de riesgo. Estos resultados son consistentes con la teoría de segmentación parcial propuesta por Errunza y Losq (1985), pero contrastan con la de Arouri (2004), quien no encuentra evidencia de que los mercados de Hong Kong y de Singapur estén segmentados con relación al mercado mundial de capitales, pues el único riesgo sistemático significativo para explicar los rendimientos en dichos 
mercados es el riesgo sistemático mundial. Se podría decir que la integración del mercado mexicano al mercado mundial de capitales sería más parecida a la de su homólogo chino, ya que la evidencia encontrada por $\mathrm{Su}$ (2000) muestra que tanto el riesgo sistemático local como el riesgo sistemático mundial son importantes para explicar los rendimientos de 27 activos enlistados en el mercado de valores de Shanghai y de 20 enlistados en el de Shenzhen.

Karolyi (2004) sugiere que las políticas económicas deficientes, la débil regulación para proteger a los inversionistas y la falta de transparencia, originan un funcionamiento deficiente de los mercados domésticos de capitales. A su vez, sostiene Karolyi, el mal funcionamiento de esos mercados puede crear incentivos para que las empresas capaces de cubrir los requisitos para enlistar sus títulos en el mercado bursátil estadounidense mediante ADR's lo hagan, en busca de mejores valuaciones y mayor liquidez. De esta forma se tiene entonces que a pesar de que esas empresas, y otras relacionadas, pueden obtener menores costos de capital, el riesgo sistemático doméstico les impide alcanzar un mínimo óptimo. En este sentido, puede concluirse que para promover el desarrollo del mercado mexicano de capitales como fuente primordial de financiamiento para las empresas locales, las autoridades mexicanas debieran realizar esfuerzos para mejorar la regulación relacionada con la preservación de los derechos de los inversionistas y de la transparencia del mercado, no solamente para continuar con otras reformas económicas.

Aunque la evidencia mostrada en este trabajo da algunas luces sobre la naturaleza multifactorial del riesgo sistemático en México y con respecto a la hipótesis de que el mercado mexicano de capitales se encuentra aún en un estado de segmentación parcial con relación al mercado mundial de capitales, deja pendientes algunos aspectos que requieren mayor estudio. Por ejemplo, es necesario avanzar en la comprensión de las características del proceso de integración del mercado mexicano de capitales con respecto al mundial, particularmente si se considera que existe suficiente soporte teórico y empírico para suponer razonablemente que el grado de integración es cambiante en el tiempo. Algo semejante podría decirse de los factores de riesgo sistemático, en tanto persista la segmentación, ante la posibilidad de modificaciones inducidas por los cambios estructurales que pudieran darse en la economía mexicana. El análisis de la relación del mercado mexicano de capitales con los de Canadá y Estados Unidos también merece atención por parte de los estudiosos, 
Riesgo sistemático en el mercado mexicano de

capitales: un caso de segmentación parcial

particularmente debido a que el TLCAN estrecha los vínculos económicos y financieros entre los tres países socios. Así pues, seguramente habrá nuevas investigaciones que vengan a enriquecer a la que se ha presentado en estas páginas.

\section{Referencias}

ALFORD, Alan. 1993. Assessing capital market segmentation: a review of the literature. En Stansell, Stanley R. (edit). International financial market integration. Blackwell. Oxford, UK.

AL-SHANFARI, Hatem. 2003. Testing the Arbitrage Pricing Theory in net oil exporting countries. Ponencia. European Applied Business Research Conference. Venice.

ALTAY, Erdinç. 2003. The effect of macroeconomic factors on asset returns: a comparative analysis of the German and the Turquish stock markets in an APT framework. Discussion Paper 48/2003. Martin Luther University. Faculty of Economics and Business Administration.

AROURI, Mohammed El Hedi. 2004. Are Stock Markets Integrated? Evidence from a Partially Segmented ICAPM with Asymmetric Effects. Working paper. University of Paris X, France.

BAILEY, Warren y Chung, Y. Peter. 1995. Exchange rate fluctuations, political risk, and stock returns: some evidences from an emerging market. Journal of Financial and Quantitative Analysis, 30 (4). 541-561.

BEKAERT, Geert y Harvey, Campbell R. 2000. Foreign speculators and emerging equity markets. Journal of Finance, 55 (2). 565-613.

; Harvey, Campbell R. y Lundblad, Christian T. 2003. Equity market liberalization in emerging markets. Journal of Financial Research, 26 (3). 275-299.

BOUDOUKH, Jacob y Richardson, Matthew. 1993. Stock returns and inflation: a long-horizon perspective. American Economic Review, 83 (5). 1346-1355. 
CABELLO, Alejandra. 1999. Globalización y liberación financieras y la Bolsa Mexicana de Valores: del auge a la crisis. Plaza y Valdés. México.

; De Jesús, Raúl y Ortiz, Edgar. 2004. Stock returns, inflation and exchange rates: Long term risk premia lags at large emerging markets. POnencia. XI International Conference, Multinational Finance Society.Istambul.Julio3-8.

CAGAN, Phillip. 1974. Common stock values and inflation: The historical record of many countries. Annual Report Supplement. NBER. New York.

CAGNETTI, Arduino. 2002. Capital Asset Pricing Model and Arbitrage Pricing Theory in the Italian Stock Market: an empirical evidence. Mimeo.

CAMPBELL, John Y. y Hamao, Yasushi. 1992. Predictable stock return in the United States and Japan: a study of long-term capital market integration. Journal of Finance, 47 (1). 43-69.

; Lo, Andrew W. y Mackinlay, A. Craig. 1997. The econometrics of financial markets. Princeton University Press. Princeton, New Jersey.

CARMICHAEL, Jeffrey y Pomerleano, Michel. 2002. The development and regulation of non-bank financial institutions. The International Bank for Reconstruction and Development/The World Bank. Washington, D.C.

CARRIERI, Francesca; Errunza Vihang R. y Hogan, Ked. 2001. Characterizing world market integration through time. Мimeo. McGill University, Montreal.

CHEN, Nai-Fu y Jordan, B.D. 1993. Some empirical tests of the arbitrage pricing theory: macrovariables vs derived factors. Journal of Banking and Finance, 17. 65-89.

; Roll, Richard y Ross, Stephen A. 1986. Economic forces and the stock market. Journal of Business, 59. 386-403.

COHN, Richard A. y Pringle, John J. 1973. Imperfections in international financial markets: implications for risk premia and the cost of capital to firms. Journal of Finance, 28 (1). 59-66. 
Riesgo sistemático en el mercado mexicano de

capitales: un caso de segmentación parcial

DARRAT, Ali F. y Zhong, Maosen. 2001. Equity market integration and multinational agreements: the Case of NAFTA. Ponencia. Annual Meeting of Financial Management International, Toronto, Canada, October 17-20.

DE LA CALLE, Luis F. 1990. Diversification of macroeconomic risk and international integration of capital markets: The case of Mexico. Mimeo. World Bank.

DOSHI, Kokila; Johnson, Robert; Ortiz, Edgar y Soenen, Luc. 2001. Privatization, liberalization and stock market performance: the case of Mexico. En Kotabe, Masaaki y Leal, Ricardo P. C. (edit.). Market revolution in Latin American: Beyond Mexico. Pergamon. Kidlington, Oxford.

DREHMAN, Mathias y Manning, Mark. 2004. Systematic factors influencing UK equity returns. Mimeo. Bank of England.

DRITSAKI-BARGIOTA, Melina y Dritsaki, Chaido. 2004. Macroeconomic determinants of stock price movements: an empirical investigation of the Greek stock market. Ponencia. XI International Conference, Multinational Finance Society. Istambul. Julio 3-8.

Emerging Stock Markets Fact Book. Varios números: 1994-2000.

ERRUNZA, Vihang R. 1979. Efficiency and the programs to develop capital markets: The Brazilian experience. Journal of Banking and Finance, 3. 355-382.

; y Losq, Etienne. 1985. International asset pricing under mild segmentation: Theory and test. Journal of Finance,. 40. 105-124.

y Miller Darius P. 2000. Market segmentation and the cost of capital in international equity markets. Journal of Financial and Quantitative Analysis, 35 (4). 577-600.

FAMA, Eugene F. 1981. Stock returns, real activity, inflation and money. American Economic Review, 71. 545-565. 
FERNANDES, Nuno. 2002. Market liberalization: spillovers from ADRs and implications for local markets. Mimeo. IESE-Escuela de Negocios. Barcelona.

GALINDO, Luís M. y Guerrero, Carlos. 1999. La transmisión de las crisis financieras: la relación entre los índices de precios de las bolsas de valores de México y Estados Unidos. Economía: Teoría y Práctica, 11. 83-95.

GILBERT, Christopher L. 1986. Professor Hendry's econometric methodology. Oxford Bulletin of Economics and Statistics, 48 (3). 283-307. Reimpreso en Granger, C. W. J. (edit.). 1993. Modelling economic series. $4^{\text {th }}$ reimp. Clarendon Press, Oxford.

GOLDBERGER, Arthur S. 1991. A course in econometrics. Harvard University Press. Cambridge, Mass. Citado en Gujarati, Damodar N. 2003. Basic econometrics. $4^{\text {th }}$ edition, McGraw-Hill Irwin. New York.

GRUBEL, Herbert G. 1968. Internationally diversified portfolios. American Economic Review, 58. 1299-1314.

HENRY, Peter Blair. 2000. Stock market liberalization, economic reform, and emerging market equity prices. Journal of Finance, 55 (2). 529-564.

HONDROYIANNIS, George y Papapetrou, Evangelina. 2001. Macroeconomic influences on the stock market. Journal of Economics and Finance, 25 (1). 33-49.

KAROLYI, G. Andrew. 2004. The role of ADRs in the development of emerging equity markets. Review of Economics and Statistics, 86 (3). 670-690.

LERICHE GUZMÁN, Cristian. 1994. Mercado de valores y financiamiento en la transición actual de la economía mexicana. En Gutiérrez Pérez, Antonio y Garrido Noriega, Celso (coord.). Transiciones financieras y TLC. Canadá, México y EEUU. Ariel. México.

LESSARD, Donald R. 1973. International portfolio diversification: a multivariate analysis for a group of Latin American countries. Journal of Finance, 28 (3). 619-633. 
Riesgo sistemático en el mercado mexicano de

capitales: un caso de segmentación parcial

LEVY, Haim y Sarnat, Marshall. 1970. International portfolio diversification of investment portfolio. American Economic Review, 60. 668-675.

LINTNER, John. 1965. The valuation of risk assets and the selection of risky investments in stock portfolios and capital budgets. Review of Economic and Statistics, 47 (1). 13-37.

LÓPEZ HERRERA, Francisco y Ortiz, Edgar. 2005. Macroeconomic risk sources: a principal component analysis for NAFTA stock markets. Ponencia. Congreso Anual de la International Trade and Finance Association. Mayo 19, Estambul, Turquía.

y Vázquez Téllez, Francisco J. 2002. Variables económicas y un modelo multifactorial para la Bolsa Mexicana de Valores: análisis empírico sobre una muestra de activos. Revista Latinoamericana de Administración, 29. 5-28.

LOTHIAN, James R. y McCarthy, Cornelia H. 2001. Equity returns and inflation: The puzzlingly long lags. Working paper. Fordham University. New York.

MADDALA, G. S. y Kim, In-Moo. 2000. Unit roots, cointegration and structural change. $2^{\text {nd }}$ reimp. Cambridge University Press. Cambridge.

MARKOWITZ, Harry M. 1952. Portfolio selection. Journal of Finance, 7 (1). 77-91.

1959. Portfolio selection, efficient diversification of investments. John Wiley \& Sons. New York.

MÁRQUEZ POZOS, Jorge Miguel; Islas Camargo, Alejandro y Venegas Martínez, Francisco. 2003. Corrientes internacionales de capital e inversión extranjera de cartera. El caso de México, 1989-1999. El Trimestre Económico, 70 (4). 791-833.

MOSSIN, Jan. 1966. Equilibrium in a capital asset market. Econometrica, 34 (4). 768-783. 
NAVA PERALTA, Noel. 1996. The Arbitrage Pricing Theory: An application for the Mexican Stock Exchange. Mimeo. Instituto Tecnológico y de Estudios Superiores de Monterrey-Campus Ciudad de México. México.

NAVARRO, Cora y Santillán, Roberto. 2001. A test of the APT in the Mexican Stock Market. Ponencia. Balas Conference. University of San Diego, San Diego.

ORTIZ, Edgar. 2000. La inversión extranjera de portafolios en los mercados de dinero y capital de México y su impacto en la crisis mexicana. En Manrique Campos Irma (coord.). Arquitectura de la crisis financiera. UNAM/IIec/ENEP AragónMiguel Ángel Porrúa. México

2004. Finanzas y productos derivados. Contratos adelantados, futuros, opciones, swaps. Mimeo. Facultad de Ciencias Políticas y Sociales. UNAM. México.

ROSS, Stephen A. 1976. The arbitrage theory of capital asset pricing. Journal of Economic Theory, 13. 341-360.

SCHOR, Adriana; Bonômo, Marco Antonio y Valls, Pereira Pedro L. 1998. Arbitrage Pricing Theory (APT) e variáveis macroeconômicas. Um estudo empírico sobre o mercado acionário brasileiro. FinanceLab Working PaperFLWP-1998-1.

SELAMAT, Zarehan. 2001. Empirical test of macroeconomic variables and stock market returns in Asian emerging markets. Mimeo. Faculty of Management, Multimedia.

SHARPE, William F. 1963. A simplified model for portfolio analysis. Management Science, 9 (2). 277-293.

1964. Capital asset prices: A theory of market equilibrium under conditions of risk. Journal of Finance, 19 (2). 425-442.

STULZ, Rene. 1981. A model of international asset pricing. Journal of Financial Economics, 9. 383-406. 
Riesgo sistemático en el mercado mexicano de

capitales: un caso de segmentación parcial

SU, Dongwei. 2000. Asset pricing in a segmented emerging market. Journal of Applied Economics, 3 (2). 387-412.

TOBIN, James. 1958. Liquidity preference as behaviour toward risk. The Review of Economic Studies, 65-86.

TREYNOR, Jack L. 1961. Market value, time and risk. Borrador no publicado. Reimpreso en French, Craig W. 2002. The Treynor capital asset pricing model. Working paper.

TREYNOR, Jack L. 1962. Toward a theory of market value of risky assets. Borrador no publicado. Una versión más acabada se publicó en Korajczyk, Robert A. (edit). 1999. Asset pricing and portfolio performance. Risk Books. Londres. 15-22.

VAN DEN GOORBERGH, Rob W. J.; De Roon, Frans A. y Werker, Bass J. M. 2003. Economic hedging portfolio. Working Paper. Finance Department and Center for Economic Research. Tilburg University. Tilburg. 